Table 1 | Selected clinical trials of TIL and related adoptive cell therapy

\begin{tabular}{|c|c|c|c|}
\hline Company or institution & Agent & Indication & Stage \\
\hline lovance Biotherapeutics & Lifileucil (LN-144) & Melanoma & Pivotal phase 2 \\
\hline lovance Biotherapeutics & LN-145 & Cervical, head and neck cancers & Phase 2 \\
\hline Netherlands Cancer Institute & TIL & Melanoma & Phase 3 \\
\hline US National Cancer Institute & Neoantigen-specific TIL & $\begin{array}{l}\text { Melanoma, breast, colorectal, ovarian, pancreatic } \\
\text { cancers; glioblastoma, }\end{array}$ & Phase 2 \\
\hline MD Anderson Cancer Center & MDA-TIL & Ovarian, pancreatic cancers; osteosarcoma, & Phase 2 \\
\hline WindMIL Therapeutics & Marrow-infiltrating lymphocytes & Multiple myeloma & Phase 2 \\
\hline H. Lee Moffitt Cancer Center & TIL & Non-small-cell lung carcinoma & Phase 1 \\
\hline
\end{tabular}

think it's this binary landscape where it's either selection or un-selection," Fardis says.

One startup, however, considers neoantigen-specific TILs very feasible today. Myst Therapeutics, founded by biomedical engineer Timothy Langer, is fully committed to TIL selection. Langer, who previously worked at Kite Pharma (now owned by Gilead) designing the Yescarta (axicabtagene ciloleucel) CAR-T product and later led development of engineered $\mathrm{T}$ cell receptors targeting neoantigens, founded Myst in January and has secured a license from the NCI. "Four or five years ago, it was perceived that even cell therapy was too difficult to do," Langer says. "Having been through that experience and looking at this particular problem, I see it as very doable, as an execution issue." Manufacturing time must be shortened, Langer says. But unlike many ideas in biotech, TIL selection in solid tumors "is one that actually has some proof of concept, or at least anecdotal proof of concept, where it has worked." Myst has raised single-digit millions from angel investors and others, and is leasing lab space at the University of California, Los Angeles.

Other biotechs and academic groups have entered the TIL space. "A lot of companies are interested in what TIL therapy may bring to the field," says Bernatchez, who says her consulting work load has grown quickly just in the past few months. TILs' curative potential is a big draw. "In melanoma, when you have a complete response with TILs that's ... over a year, then usually the patient is cured," Bernatchez says. Of the NCI's 46 complete responders in melanoma, says Goff, only two have relapsed. Non-melanoma solid tumors will be harder, and success or failure there will determine whether TILs remain a niche treatment or evolve into a foundational cancer therapy.

\section{Ken Garber}

Ann Arbor, Michigan, USA

Published online: 7 August 2019

https://doi.org/10.1038/d41587-019-00023-6

\title{
PODCAST
}

\section{First Rounders: Nina Tandon}

Nina Tandon is cofounder and CEO of EpiBone. Her First Rounders conversation covers growing up on Roosevelt Island in New York City, witnessing September 11, and tackling her company's first phase 1 trial. https://www.nature.com/nbt/podcast

Published online: 4 September 2019

https://doi.org/10.1038/s41587-019-0243-7

"It's like saying a skateboard needs to have a sticker to say it can't be used on the freeway. It doesn't make any sense." Josiah Zayner, biohacker and founder of Odin, a company providing do-it-yourself kits for genetic engineers, known for injecting himself with CRISPR on social media, comments on a new law in California prohibiting the sale of CRISPR kits unless they warn against self-administration. (MIT Technology Review, 9 August 2019)
"Manufacturing pharmaceuticals is difficult, painstaking, and dangerous. If you get the dosing or the strength on insulin wrong, it's death. If you let contaminants into the insulin, it's possible death. If your insulin breaks down too quickly in storage, it's death." Stanford's Hank Greely comments on the 'Open Insulin Project', run by a group of biohackers in Oakland, California, to create a low-cost insulin. (Dw.com, 24 May 2019)

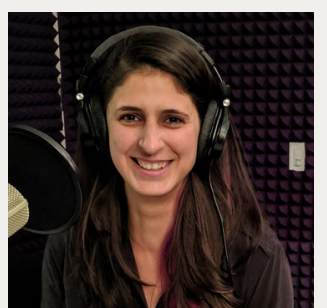

"Consumers might be surprised by some of the uses that their data are being put toward, and they might be concerned about some of the third parties their data are being shared with." James Hazel of Vanderbilt's Center for Genetic Privacy and Identity in Community Settings, in light of Congress considering legislation on digital data privacy that could restrict what direct-to-consumer genomics companies can do with the data they collect. (STAT, 9 August 2019) 